\title{
WG3 Highlights: Electroweak Physics and Beyond the Standard Model
}

\author{
Maxime Gouzevitch ${ }^{1 *}$ \\ ${ }^{1}$ Université Claude Bernard-Lyon I, 69622 Villeurbanne Cedex, France \\ E-mail: mgouzevi@cern.ch
}

\section{Anna Kaczmarska ${ }^{2}$}

${ }^{2}$ IFJ PAN, 31-342 Kraków, Poland

E-mail: anna.kaczmarska@ifj.edu.pl

\section{Margarete Mühlleitner ${ }^{3}$}

${ }^{3}$ Karlsruhe Institute of Technology (KIT), 76131 Karlsruhe, Germany

E-mail: milada.muehlleitnerekit.edu

\section{Krzysztof Turzynski ${ }^{4}$}

${ }^{4}$ University of Warsaw, 00-927 Warsaw, Poland

E-mail: krzysztof-jan.turzynski@fuw.edu.pl

\begin{abstract}
The working group on electroweak physics and beyond the Standard Model (SM) physics at the Deep Inelastic Scattering 2014 workshop included results ranging from Higgs over gauge boson physics to searches for supersymmetric particles and exotic states, performed by the Large Hadron Collider (LHC) experiments ATLAS and CMS. Higgs results from the Tevatron experiment have been presented as well. Each session was complemented by invited theoretical overviews on the subject under discussion. We give a short summary by presenting some selected results. For more details we refer to the individual contributions collected in these proceedings.
\end{abstract}

XXII. International Workshop on Deep-Inelastic Scattering and Related Subjects, 28 April - 2 May 2014

Warsaw, Poland

\footnotetext{
${ }^{*}$ These proceedings summarize the results available at the time of the conference.
} 


\section{Introduction}

The discovery of the Higgs boson by the LHC experiments ATLAS and CMS [1, 2] entailed an intense experimental activity in order to determine the properties of the discovered particle. Although the Higgs boson looks very SM-like, there is still room for interpretations within theories beyond the SM (BSM), especially that the dark matter (DM) content of the Universe can only be understood in terms of BSM physics. While so far no direct sign for new physics (NP) has been detected the experiments continue their search for supersymmetric (SUSY) particles and exotic states, in more and more refined analyses. The SUSY searches not only cover the search for coloured sparticles, the squarks and gluinos, but also for the electroweak SUSY partners. In the exotic sector a strong experimental activity pushes up the limits for new particles. Indirect hints for BSM physics may arise from the measurements in the Higgs, gauge and top sectors. With the accumulation of data and the development of new experimental strategies, the measurements of the Higgs properties become more and more precise. Thus first evidence for the Higgs couplings to fermions has been provided. Moreover, an upper limit on the total width of the Higgs boson has been given that is four times the SM width, an accuracy that would have been thought impossible to be achieved before the start of the LHC. In the gauge sector the experimental results allow for consistency tests and the search for anomalous triple and quartic couplings. Top physics has been covered in a joint session together with working group 5 on Heavy Flavours, to which we refer for more details.

\section{Higgs Physics}

The investigation of the properties of the discovered scalar particle not only allows to establish it as the Higgs boson. In case of deviations from the SM properties the Higgs measurements also give indirect hints to beyond the SM physics. Thus the precise determination of the Higgs couplings allows for tests beyond the scales that are directly reachable at the experiment and/or complements direct searches [3]. The Higgs boson mass, which in the SM is a fundamental parameter and not given by the theory, has to be measured at highest possible accuracy, as it feeds back in the uncertainty on the SM Higgs couplings. Furthermore, it allows for a self-consistency test of the SM at the quantum level, and its value is related to the stability of the electroweak vacuum [4]. In BSM theories, in which the Higgs mass value can be calculated, its knowledge can be exploited to test parameter relations, see e.g. [5] for a recent discussion. Using the data sample collected at the c.m. energies of 7 and $8 \mathrm{TeV}$, corresponding to an integrated luminosity of $25 \mathrm{fb}^{-1}$, ATLAS has provided an improved measurement of the Higgs boson mass derived from a combined fit to the invariant mass spectra of the Higgs decays into photon pairs and into $Z Z^{*}$, which subsequently decay into four leptons. The measured value of the Higgs boson mass is $m_{H}=125.36 \pm 0.37$ (stat) \pm 0.18 (syst) GeV [6], $c f$. Fig. 1 (left). Based on the data samples collected at $7 \mathrm{TeV}$ c.m. energy, corresponding to an integrated luminosity of $5.1 \mathrm{fb}^{-1}$, and at $8 \mathrm{TeV}$ with $19.7 \mathrm{fb}^{-1}$, CMS measures the mass of the Higgs boson to be $m_{H}=125.03_{-0.27}^{+0.26}$ (stat) ${ }_{-0.15}^{+0.13}$ (syst) from the high-resolution $\gamma \gamma$ and $Z Z$ channels [7], Fig. 1 (right).

A Higgs boson with mass around $125 \mathrm{GeV}$ allows for decays into many different SM final states. The fits to the experimentally extracted signal strength parameters give access to the Higgs 

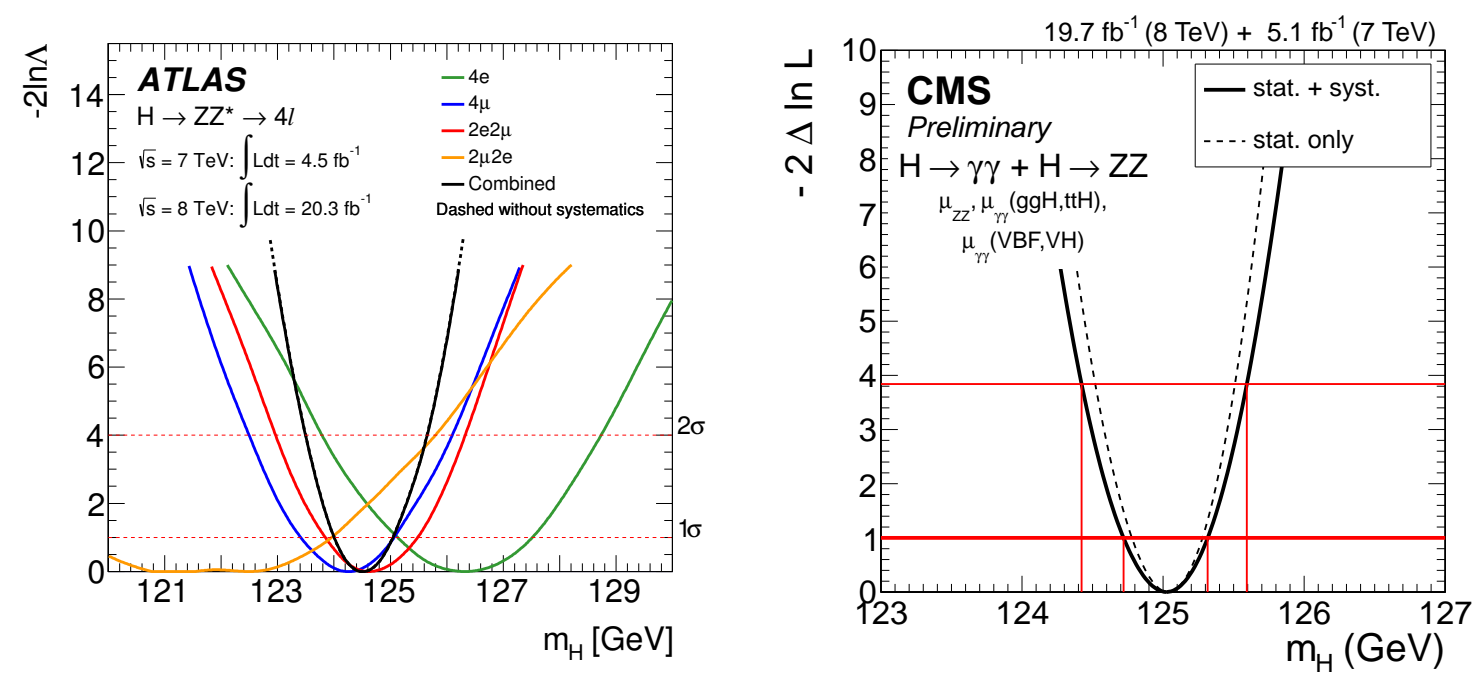

Figure 1: Left: Value of $-2 \ln \Lambda$ reported by the ATLAS experiment as a function of $m_{H}$ for the individual $H \rightarrow \gamma \gamma$ and $H \rightarrow Z Z^{*} \rightarrow 4 l$ channels and their combination. For details, see [6]. Right: Scans of the test statistics $q\left(m_{H}\right)$ versus $m_{H}$ for the combination of the $\gamma \gamma$ and $4 l \mathrm{CMS}$ analyses. For details, see [7].

boson couplings. The strategy that is presently adopted is to assume, that the signal arises from a single resonance, that the narrow width approximation can be applied, to decouple production and decay, and finally, that the tensor structure is the same as in the SM and only the coupling strength is allowed to be modified [8]. The improvements on the coupling measurements since the Higgs boson discovery show an increasing consistency with the SM predictions. The couplings to the gauge bosons, $\gamma \gamma, Z Z$ and $W W$, have been established. The experiments also have first evidence for Higgs decays into fermions. The fermionic decays are more difficult to access due to large backgrounds or small branching ratios. The combination of the CMS results in the $H \rightarrow$ $b \bar{b}$ (see also Fig. 2 (left)) and $H \rightarrow \tau \tau$ channels under the assumption of SM Higgs production, reveals strong evidence for the direct coupling of the Higgs boson to down-type fermions with an observed (expected) significance of 3.8 (4.4) standard deviations [10]. The ATLAS experiment finds evidence for the direct decay into fermions at the $3.7 \sigma$ level from the combination of the $H \rightarrow$ $b b$ and $H \rightarrow \tau \tau$ channels with a signal strength of $\mu_{b b, \tau \tau}=1.09 \pm 0.24$ (stat) ${ }_{-0.21}^{+0.27}$ (sys) $[11,12,13]$, cf. e.g. Fig. 2 (right). Upper limits on the decays into $\mu \mu$ have been given, showing no significant excess over the background $[14,15]$. Their further investigation will be interesting at the next run of the LHC and its high-luminosity option. The associated production of a Higgs boson with a $t \bar{t}$ pair directly probes the top Yukawa coupling. Because of the small cross section and large backgrounds, it is difficult to access. The CMS combination of all $t \bar{t} H$ channels results in an observed 95\% CL upper limit of 4.3 on the signal strength modifier $\mu=\sigma / \sigma_{\mathrm{SM}}$ for $m_{H}=125.7 \mathrm{GeV}$ [16]. In the $t \bar{t} H \rightarrow b \bar{b}$ channel ATLAS reports an observed 95\% CL upper limit on the signal strength of 4.1 times the SM expectation for $m_{H}=125 \mathrm{GeV}$ [17].

Due to the smallness of the SM Higgs total width for a $125 \mathrm{GeV}$ Higgs boson, $\Gamma \approx 4.4 \mathrm{MeV}$, it cannot be accessed via a lineshape measurement. The CMS experiment has presented first constraints on the total width, using the on-shell and off-shell Higgs production and decay into $Z Z$ 

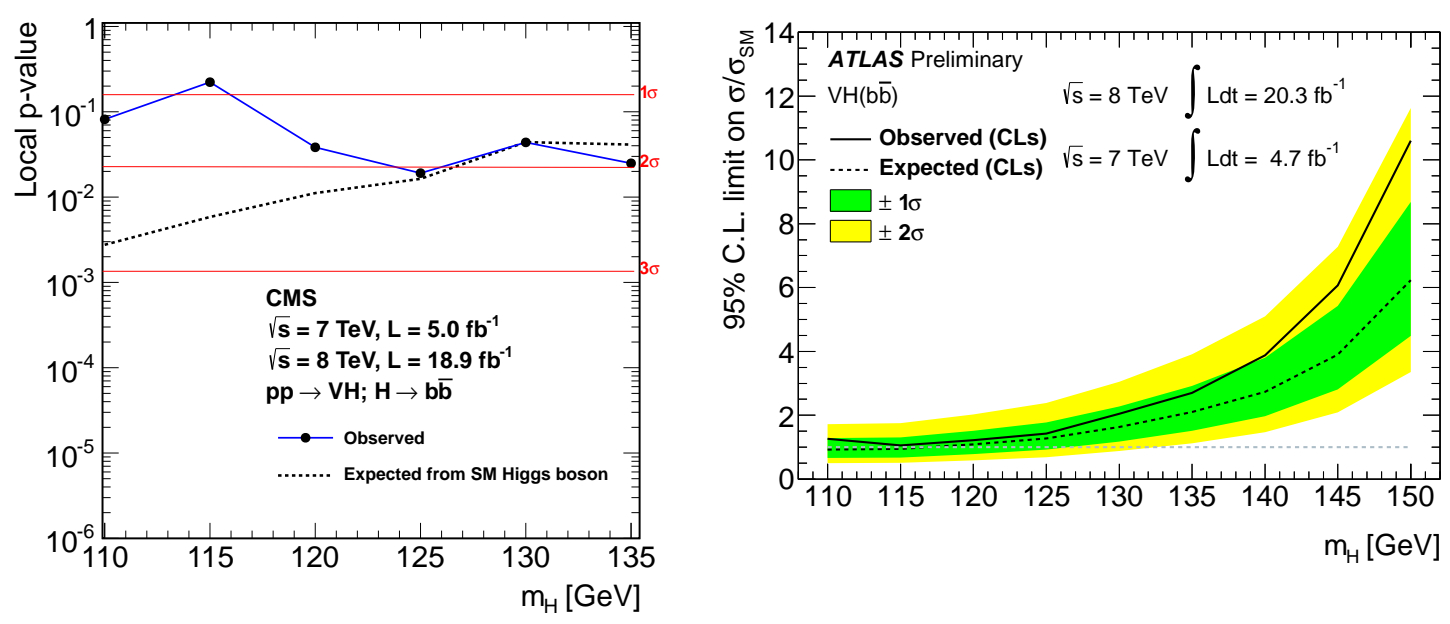

Figure 2: Left: Local p-values and corresponding significance (measured in standard deviations) for the background-only hypothesis to account for the observed excess of events in the CMS data. Details can be found in [9]. Right: ATLAS expected (dashed) and observed (solid) 95\% confidence level $V H(b \bar{b})$ cross section upper limits, normalised to the SM Higgs boson production cross section, as a function of $m_{H}$ for all channels and data taking periods combined, details given in [11].

[18]. Based on the data collected at $\sqrt{s}=7$ and $8 \mathrm{TeV}$ with, respectively, $5.1 \mathrm{fb}^{-1}$ and $19.7 \mathrm{fb}^{-1}$ integrated luminosity, an upper limit on the Higgs boson width of $\Gamma_{H}<22 \mathrm{MeV}$ at $95 \%$ confidence level (CL) has been derived, which is 5.4 times the expected SM value at $m_{H}=125.6 \mathrm{GeV}$, cf. Fig. 3 (left). ${ }^{1}$

The CMS and ATLAS collaborations also have performed measurements in the context of BSM Higgs physics. Thus ATLAS and CMS presented results on the search for neutral Higgs bosons of the Minimal Supersymmetric Extension of the SM (MSSM) [20, 21, 22], on interpretations within the 2 Higgs Doublet Model Extension (2HDM) of the SM [23, 24, 25], on searches for light pseudoscalars of the Next-to-Minimal Superysymmetric Extension of the SM (NMSSM) [26, 27] and on charged Higgs searches [28, 29, 30, 31]. ATLAS showed results for doubly charged Higgs boson searches [32], on an invisibly decaying Higgs boson [33] and for the flavour changing neutral current (FCNC) decay $t \rightarrow c H(\gamma \gamma)$ [34]. The CMS collaboration reported their findings in the context of an SM extension with a singlet field [35], on rare Higgs decays into $Z \gamma$ [36], on $H \rightarrow \gamma^{*} \gamma \rightarrow \mu \mu \gamma$ [37] and on invisible Higgs decays [38]. New results have been shown on the search for the resonant production of Higgs pairs in the decay channel $X \rightarrow H H \rightarrow \gamma \gamma b b$ at $\sqrt{s}=8 \mathrm{TeV}$ using $19.7 \mathrm{fb}^{-1}$ integrated luminosity [39]. For the investigated mass range between $m_{X}=260$ and $1100 \mathrm{GeV}$ upper limits at $95 \% \mathrm{CL}$ on the cross section between about 4 and $0.4 \mathrm{fb}$ have been extracted, $c f$. Fig. 3 (right). ${ }^{2}$ Neither ATLAS nor CMS find any deviations from the Standard Model predictions in the BSM Higgs searches. While this narrows down the parameter

\footnotetext{
${ }^{1}$ In the meantime also ATLAS provided an observed (expected) $95 \%$ CL upper limit on $\Gamma_{H} / \Gamma_{H}^{\mathrm{SM}}$ in the range 4.8-7.7 (7.0-12.0) [19].

${ }^{2}$ ATLAS has recently published results on resonant and non-resonant Higgs boson pair production in the $h h \rightarrow \gamma \gamma b \bar{b}$ final state at a c.m. energy of $8 \mathrm{TeV}$ and an integrated luminosity of $20.3 \mathrm{fb}^{-1}$ [40].
} 

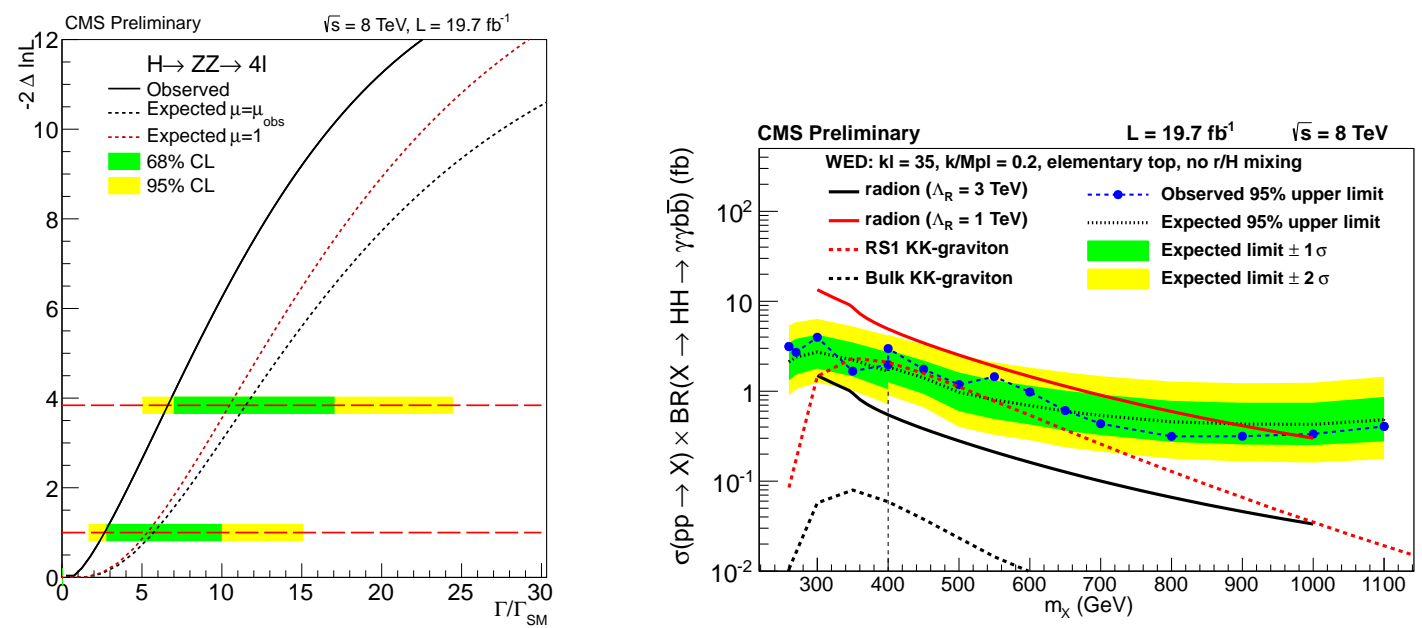

Figure 3: Left: Likelihood scan of $\Gamma / \Gamma_{\mathrm{SM}}$, details given in [18]. Right: Expected and observed 95\% CL upper limits on the cross section times branching ratios $\sigma(p p \rightarrow X) \times B R(X \rightarrow H H \rightarrow \gamma \gamma b b)$, from [39].

space for possible BSM extensions, there is still plenty of room for a variety of new physics models that are compatible with the Higgs results. This has been exemplified for the parameter space of the 2HDM [41], where two regions are still allowed, one corresponding to the SM limit and the other one given by the wrong-sign scenario with Higgs couplings to down-type quarks having opposite sign compared to the couplings to massive vector bosons.

Various spin-parity hypotheses have been tested by the LHC experiments and have been excluded in favor of the SM $J^{P}=0^{+}$hypothesis $[42,43,44]$. The techniques used in the determination of the Higgs quantum numbers can also be exploited to test for Higgs anomalous couplings as pointed out in [45].

The last step in the experimental verification of the Higgs mechanism will be the determination of the Higgs self-couplings [46]. Although the determination of the trilinear Higgs self-coupling in Higgs pair production at the LHC will be challenging, a lot of activity has started to investigate possible strategies to extract this coupling from di-Higgs production, see [47] and references therein. The measurement will certainly require high luminosity.

From the theoretical point of view the interpretation of the Higgs results in a rather modelindependent approach can be performed by the introduction of an effective Lagrangian, in which the leading effects beyond the SM Lagrangian arise at the dimension-6 level. There has been going on a quite intense discussion on what are the operators that constrain Higgs physics, see e.g. [3, 48] and references therein. At this workshop, the parametrisation in terms of BSM primary effects was presented, which allow to constrain all possible interactions at the dimension-6 level [49]. There are 8 primaries related to Higgs physics, 3 related to triple gauge couplings and 7 related to $Z$-pole measurements at LEP. The Lagrangian can be used to derive the constraints on all BSM processes arising at the dimension- 6 level from the BSM primaries. 

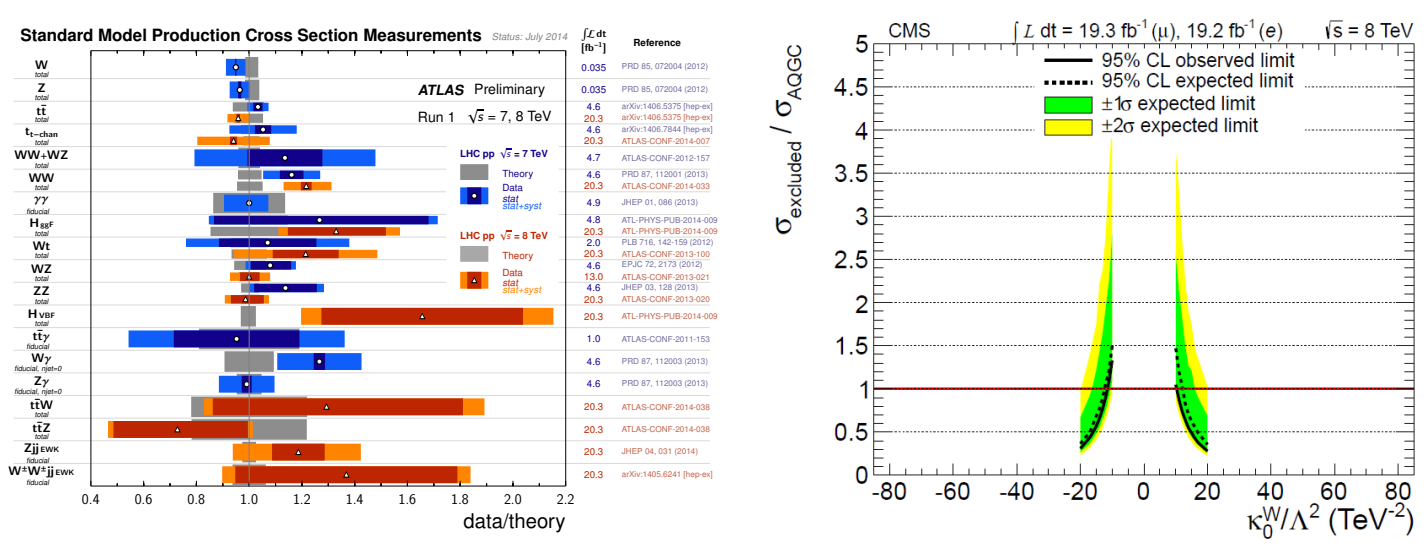

Figure 4: Left: The data/theory ratio for several Standard Model total and fiducial production cross section measurements performed by ATLAS, corrected for leptonic branching fractions. All theoretical expectations were calculated at NLO or higher. Details are given in [50]. Right: Exclusion limits for the parameter $\kappa_{0}^{W}$ at 95\% CL, using the photon $p_{T}$ as observable, see [56] for details.

\section{Electroweak Physics}

In the session dedicated to gauge boson physics talks were presented on single and multi-boson production in QCD and electroweak (EW) processes as well as the measurement of a rare decay channel $Z \rightarrow 4 l$ cross section.

Di-boson $(W / Z \gamma, W W, W Z, Z Z)$ production at the LHC is of great importance as it allows for fundamental tests of the electroweak sector, probes New Physics through anomalous triple gauge couplings and constitutes an irreducible background for the Higgs searches. Studies of di-bosons from Higgs decays on the other hand give access to the Higgs properties, its couplings to gauge bosons, its mass and width as well as to its spin-parity quantum numbers, $c f$. Sec. 2. The diboson search is performed by looking for an excess at high di-boson invariant mass (when it can be reconstructed) or boson transverse momentum. The di-boson data from the LHC and Tevatron experiments are in general consistent with (next-to-)next-to-leading order ((N)NLO) SM predictions as shown for example in Fig. 4 (left), and new stringent limits are set on the contributions from effective dimension-6 operators. However in some channels such as $W^{+} W^{-}$production discrepancies of up to 2 standard deviations started to appear when the precision of the measurements increased (typically to better than $10 \%$ for the total cross section) [51]. Therefore, in order to reach the accuracy achieved in the experiment and to be able to properly interpret the experimental data, the theory predictions for the processes must be as precise as possible. Three theory contributions reported on the status and the progress on vector boson pair production [52], multi-boson production in weak boson fusion [53] and $W^{+} W^{-}$production via subleading processes [54]. In particular the impact of the NLO EW corrections was predicted to reach up to $20 \%$ in the energy range that corresponds to the highest momentum scales accessible at $8 \mathrm{TeV}$ at the LHC [52].

A particularly interesting channel for the understanding of the electroweak symmetry breaking (EWSB) mechanism is the production of the Higgs boson through vector boson fusion. The process is characterized by the presence of two forward-backward quark jets with low hadronic activity in 
between (rapidity gap). The properties of this process were studied in a similar environment by measuring the EW production of the $Z$ boson [55].

Vector boson scattering (VBS) processes require high luminosity $\left(\mathscr{O}\left(100 \mathrm{fb}^{-1}\right)\right)$ and a high c.m. energy for a precise investigation. Di-boson production through VBS and the tri-boson production are sensitive to the non-Abelian structure of the weak interaction and to the unitarisation mechanism at high di-boson mass related to the EWSB mechanism. With the data collected at 7 and $8 \mathrm{TeV}$ first insights can be gained, however, and a search for the quartic anomalous gauge couplings can be performed. First experimental limits on the dimension- 8 parameters parametrising anomalous $W W \gamma \gamma$ and $W W Z \gamma$ quartic gauge couplings have been set by CMS in the search for $W W \gamma$ and $W Z \gamma$ production [56], $c f$. Fig. 4 (right).

\section{Supersymmetry}

One of the motivations for SUSY is the possible solution of the hierarchy problem which entails SUSY scales that are in reach of the LHC. In particular coloured sparticles, gluinos and squarks, are copiously produced in $p p$ collisions. Until now, no discovery of SUSY particles has been announced, and the experiments have started looking for signatures from compressed spectra, events with higher jet and lepton multiplicity and for long-lived particles, with the aim to investigate all possible corners of the parameter space in order not to miss any sign of new physics. The SUSY searches can be divided in signatures originating from R-parity conserving and R-parity violating (RPV) theories. In case of R-parity conservation the SUSY particles are produced in pairs and decay into the lightest SUSY particle (LSP) which is stable. These events are characterized by missing transverse energy $E_{T}$ and many high transverse momentum, $p_{T}$, jets and leptons. In Rparity violating set-ups on the other hand the SUSY particles can be created singly without missing $E_{T}$ final states. Depending on the produced sparticle the decay chains can be long or short and involve different multiplicities of jets and leptons. The searches are categorized according to the number of jets and leptons in the final states. The strategy for the estimation of the background is to rely as little as possible on simulations and to exploit data-driven methods. For the latter control regions are defined where little or no signal is expected, the background is measured in these control regions and extrapolated to the signal region. Due to the large number of parameters in SUSY theories, a model-independent search is not possible. Instead the results are interpreted within specific models like e.g. the minimal supergravity (mSUGRA) inspired constrained MSSM (CMSSM), the phenomenological MSSM (pMSSM) or simplified models (SMS).

The objects that ATLAS uses in its inclusive searches for squarks and gluinos are jets, $b$ tagged jets, leptons, taus, photons and missing $E_{T}$. New results were reported on the search for multi-jet events with exactly two same-sign leptons or at least three leptons, using data collected at $\sqrt{s}=8 \mathrm{TeV}$ and corresponding to an integrated luminosity of $20.3 \mathrm{fb}^{-1}$ [57]. In order to extend the sensitivity also $b$-quark jets, missing $E_{T}$ and other observables were utilized. No deviations from the SM were observed and new or significantly improved exclusion limits were set on various SUSY models with the lightest squark being of the first, second or third generation, allowing for R-parity conservation or violation. See e.g. Fig. 5 (left) for the exclusion limits placed on direct sbottom production in a simplified model interpretation. In another newly presented search [58] with diphoton events and large missing $p_{T}$ at $\sqrt{s}=8 \mathrm{TeV}$ and $\int \mathscr{L}=20.3 \mathrm{fb}^{-1}$, besides model- 

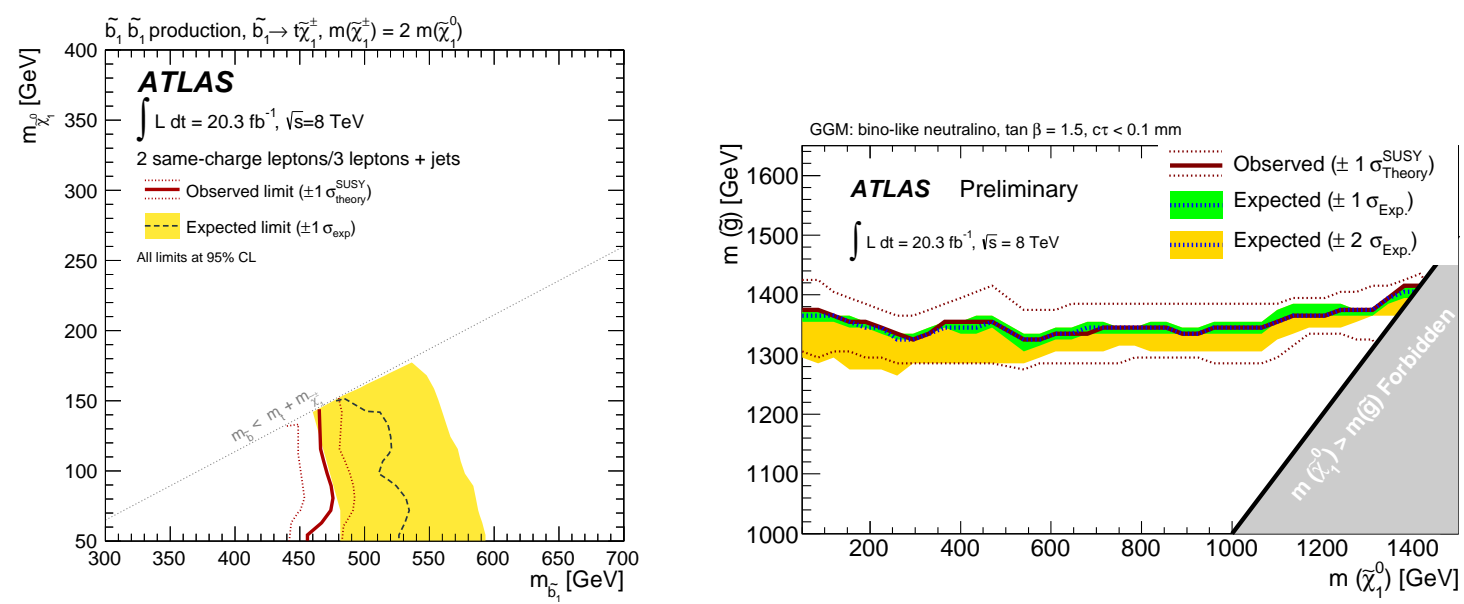

Figure 5: Left: Observed and expected exclusion limits on direct sbottom production with $m_{\tilde{\chi}_{1}^{0}}=m_{\tilde{\chi}_{1}^{ \pm}} / 2$, [57]. Right: Expected and observed 95\% CL lower limits on the gluino mass as a function of the neutralino mass in the GGM model with a bino-like lightest neutralino NLSP, from [58].

independent limits, lower limits were set on the gluino and wino masses under the hypothesis of general gauge mediation (GGM), $c f$. Fig. 5 (right). In [59] a model-independent approach was presented, which exploits topologies involving isolated electrons, photons and muons, as well as jets, including $b$-jets and missing $p_{T}$. Based on 697 classes with a Standard Model expectation greater than 0.1 events at $\sqrt{s}=8 \mathrm{TeV}$ and a total integrated luminosity of $20.3 \mathrm{fb}^{-1}$, no significant deviation from the SM has been found.

The CMS inclusive searches are characterized by final states involving 0-lepton, 1-lepton, 2lepton, multi-lepton and photon signatures. The data sample collected at $\sqrt{s}=8 \mathrm{TeV}$, corresponding to an integrated luminosity of $19.5 \mathrm{fb}^{-1}$ has been divided into three jet multiplicity categories with 3-5, 6-7 and $\geq 8$ jets [60]. Extending the SUSY parameter space explored by searches in the all-hadronic final state, the observed numbers of events are consistent with the expected SM background evaluated from the data. The results are interpreted in the context of simplified models, and squark masses below $780 \mathrm{GeV}$ as well as gluino masses of up to $1.1-1.2 \mathrm{TeV}$ are excluded at 95\% CL for LSP masses below $100 \mathrm{GeV}$, as shown in Fig. 6.

The cross sections for chargino, neutralino and slepton production at the LHC are smaller than for coloured sparticle production, but lead to clean signatures. From the CMS collaboration results were presented on the search for electroweak charginos, neutralinos and sleptons using leptonic final states [61], chargino and neutralino production in final states with a Higgs boson [62] and finally EW higgsino production in channels with two Higgs bosons decaying into $b$ quarks [63]. All results are based on data samples recorded at a c.m. energy of $8 \mathrm{TeV}$ corresponding to integrated luminosities of $19.3 \mathrm{fb}^{-1}$ to $19.5 \mathrm{fb}^{-1}$. In the search with $4 b$ in the final state [63], due to a slight excess in the observed number of events compared to the estimated background no exclusion for any higgsino mass value is obtained, $c f$. Fig. 7. The ATLAS experiment has exploited 1 lepton $(+b b), 2$ lepton/2 $\tau, 3$ lepton $(e, \mu, \tau)$ and 4 lepton signatures to search for charginos, neutralinos and sleptons, in decay chains that can be $W, Z$ or Higgs-mediated as well as slepton, respectively, 

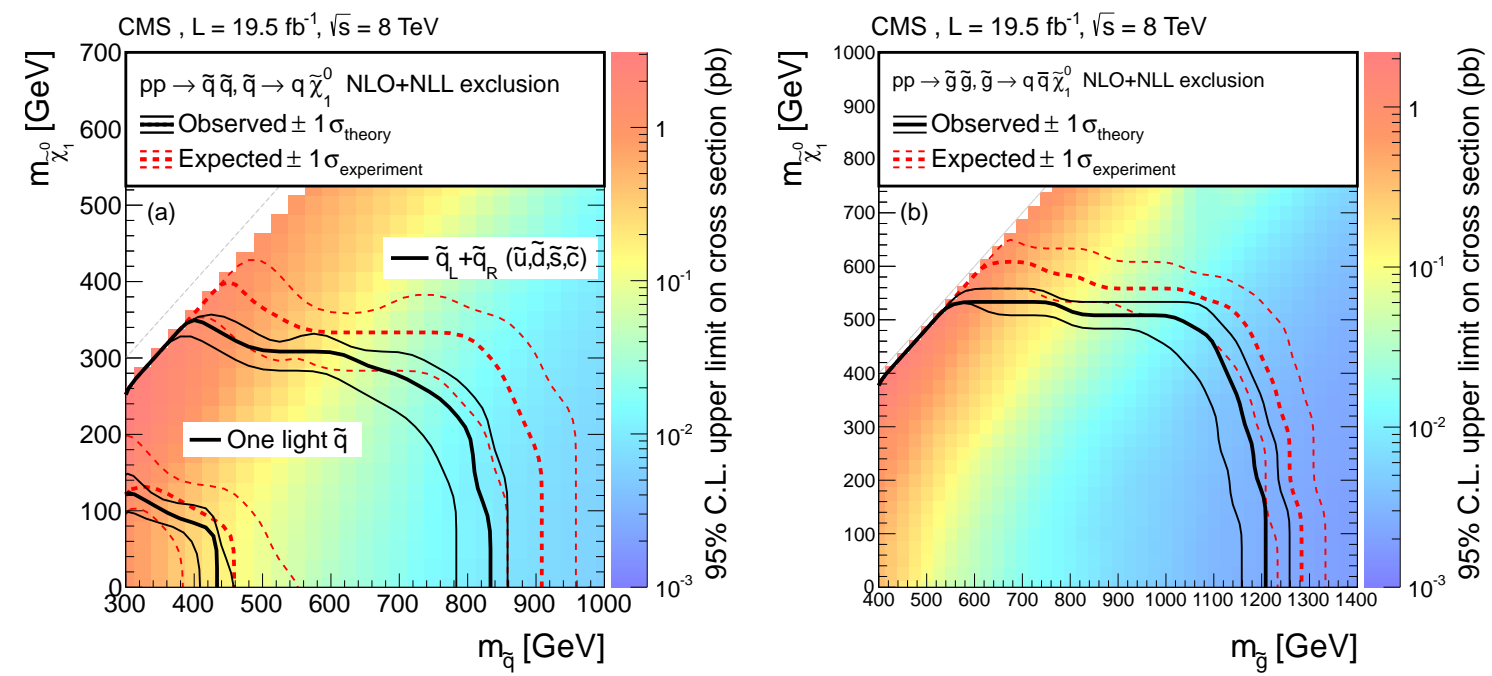

Figure 6: The observed and expected $95 \% \mathrm{CL}$ upper limits on the (left) $\tilde{q} \tilde{q}$ and (right) $\tilde{g} \tilde{g}$ production cross sections in either the $\left(m_{\tilde{q}}, m_{\tilde{\chi}_{1}^{0}}\right)$ or the $\left(m_{\tilde{g}}, m_{\tilde{\chi}_{1}^{0}}\right)$ plane obtained with the simplified models, Fig. from [60] .

stau mediated. In [64] limits were set on the masses of the lightest chargino, the next-to-lightest neutralino and sleptons ( $c f$. Fig. 7 (right)) for different lightest-neutralino mass hypotheses in SMS based on searches in final states with two leptons (electrons and muons) and missing $p_{T}$ using $20.3 \mathrm{fb}^{-1}$ of data at $\sqrt{s}=8 \mathrm{TeV}$.

Third generation squarks are interesting as due to large mixing they can be substantially lighter than the remaining squarks. They can be produced either directly in $p p$ collisions or from gluino decays. Both ATLAS and CMS have presented a variety of searches for stops and sbottoms. No excess in the data with respect to the SM expectations has been observed and exclusion limits have
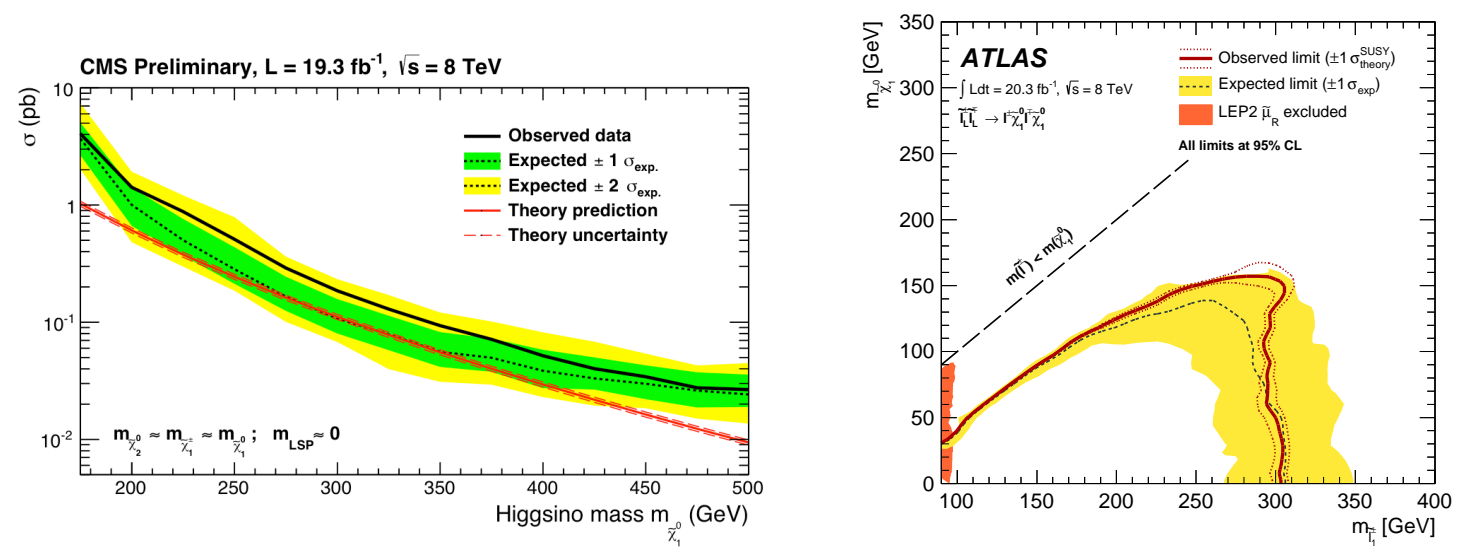

Figure 7: Left: Observed and expected upper limits on the cross section as a function of higgsino mass, details given in [63]. Right: 95\% CL exclusion regions in the $m_{\tilde{\chi}_{1}^{0}}-m_{\tilde{l}}$ plane for left-handed selectron and smuon production, from [64]. 
been placed. Still stops can be rather light, in particular in scenarios where the stop is so light that it decays via the flavour-violating two-body decay into charm and the lightest neutralino $[65,66]$ or via the four-body decay into a bottom quark, the lightest neutralino and a fermion pair [67]. A summary on the limits on third generation squarks reported by ATLAS and CMS in sample final states is shown in Figs. 8 (left) and (right).
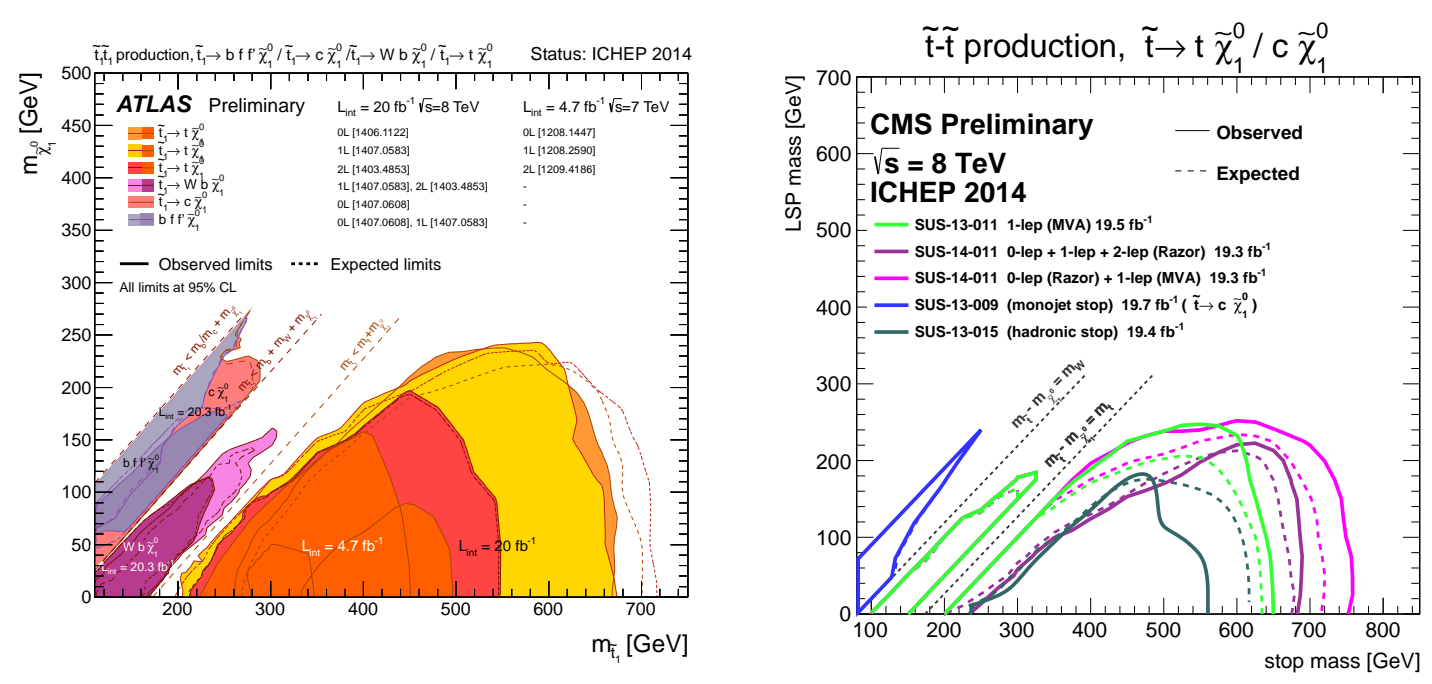

Figure 8: Left: ATLAS 95\% CL exclusion regions in the $m_{\tilde{\chi}_{1}^{0}}-m_{\tilde{t}_{1}}$ plane, for details see [68]. Right: CMS 95\% CL exclusion regions in the $m_{\tilde{\chi}_{1}^{0}}-m_{\tilde{t}_{1}}$ plane, in the $\tilde{t} \rightarrow t \tilde{\chi}_{1}^{0}$ and $\tilde{t} \rightarrow c \tilde{\chi}_{1}^{0}$ decays, from [69].

Giving up the assumption of R-parity conservation, the LSP can decay into SM particles. The final states are then characterized by multi-leptons and multi-jets without missing $E_{T}$ signatures. In case of very small couplings the LSP can have a long lifetime. Also in R-parity conserving models long lifetimes are possible, e.g. for a gluino if the squarks mediating its decay are heavy, or in gauge-mediated SUSY breaking (GMSB) scenarios, where a weak coupling between the next-tolightest SUSY particle and the gravitino can lead to stable sleptons or delayed photons. Two main classes of particles exist: lepton-like or hadron-like particles. The latter are color-charged and hadronize to form R-hadrons with quarks or gluons. The signatures for stable massive particles involve slowly moving particles with high momentum, anomalously high ionization energy loss and a large time-of-flight. They may lead to displaced vertices or disappearing tracks. Both LHC experiments have probed a wide range of RPV and long-lived particle signatures. No excess over the SM has been observed.

In order to properly interpret the SUSY searches and derive reliable exclusion limits, the theory community must provide precise predictions for SUSY particle production and decay, including higher order corrections, not only for inclusive quantities, but also for differential distributions. Results on squark pair and squark-antisquark production at NLO including the full mass dependence and combined with NLO decays into quarks and neutralinos at the fully differential level have been presented at this workshop and shown that differential $K$-factors cannot in general assumed to be flat [70]. Taking finally the entity of the LHC results on Higgs and SUSY physics (together with results from DM measurements) and interpreting them within various BSM scenarios, they can 
provide quite relevant constraints on viable NP scenarios. Two such interpretations were presented during the session. By performing multi-parameter scans over the MSSM parameter space, the authors reported new regions consistent with the existing data and characterized by a moderately heavy higgsino or bino-higgsino mixed neutralino [71] or a light bino [72] as the DM candidate.

\section{Exotic Physics}

Besides supersymmetry there exists a plethora of NP extensions that address different aspects of the flaws of the SM like e.g. the hierarchy problem or the absence of a viable DM candidate. Using the data collected at the 7 and $8 \mathrm{TeV}$ run of the LHC corresponding to $\mathscr{O}\left(20 \mathrm{fb}^{-1}\right)$, the LHC collaborations have examined a series of signatures and interpreted them in the context of various exotic NP models. No significant deviation from the SM expectations has been found and limits have been placed. Thus searches have been conducted for heavy resonances in di-lepton and lepton plus missing $E_{T}$ channels, for excited quarks in $\gamma$ plus jets final states and for leptoquarks leading to bottom plus $\tau$ and top plus $\tau$ signatures. Another class of analyses investigates resonant di-boson production, where the well known $W / Z$ boson masses and the good kinematic reconstruction can be exploited to suppress backgrounds. They allow to place upper limits e.g. on models with an extended gauge sector, on SMS with heavy vector triplets, on techni-hadrons, on neutral resonances as they appear e.g. in bulk graviton or Randall-Sundrum models. The combination of the leptonically decaying gauge bosons with the all-hadronic channel leads to the exclusion limits shown in Fig. 9 (left). Searches with mono-jets, mono-photons or mono- $W / Z$ 's allow for interpretations in
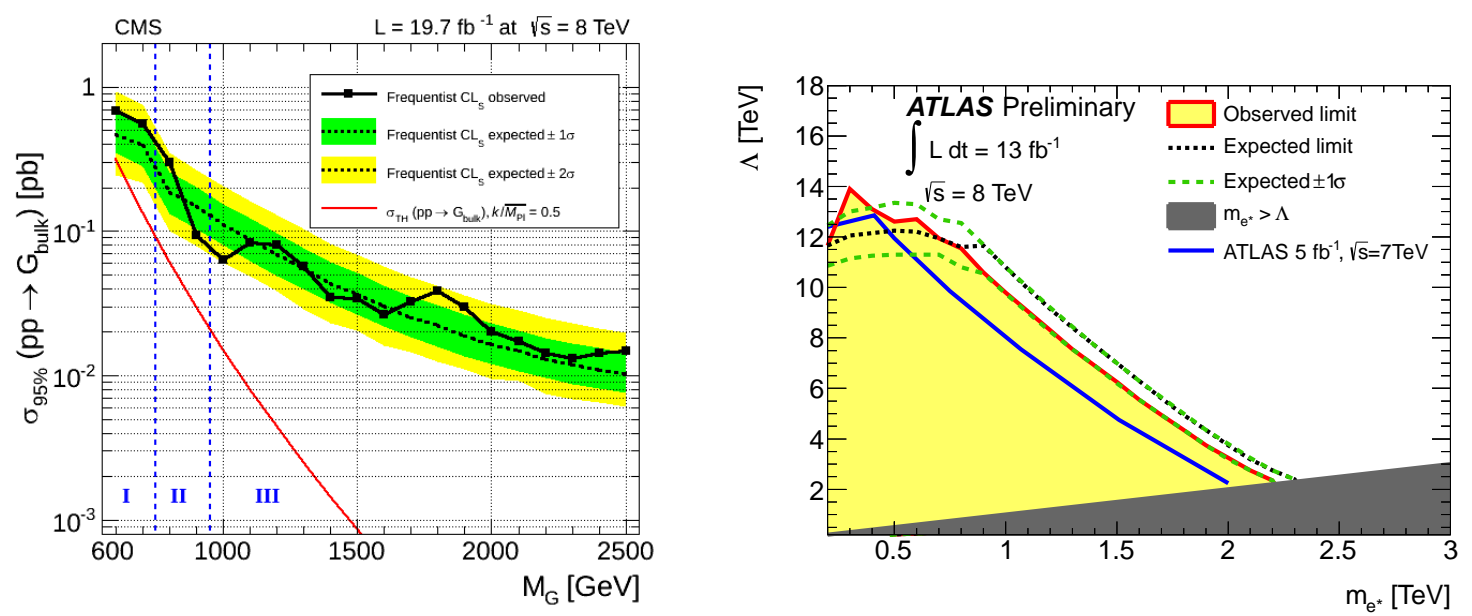

Figure 9: Left: Observed (solid) and expected (dashed) 95\% CL upper limit on the cross section from the production of a bulk graviton decaying into boson pairs in their leptonic final states combined with the all-hadronic channel [73]. Right: ATLAS exclusion limits in the compositeness scale $(\Lambda)$ versus the excitedlepton mass $\left(m_{l^{*}}\right)$ parameter space for the electron channel. The filled area is excluded at 95\% CL. No limits are set in the dark shaded region $m_{l^{*}}>\Lambda$ where the model is not applicable. Figure from [74].

the context of e.g. DM production, large extra dimension models, supersymmetry or Higgs decays into invisible particles. While no significant excess has been observed, the limits on the various 
models have been improved. Exploiting new reconstruction techniques for boosted top quarks, ATLAS and CMS have performed many searches for resonances decaying into $t \bar{t}$ or $b t$ as well as for vector-like quarks entailing tops or bottoms in their decays. Searches for BSM physics in events with multiple leptons finally, can test left-right symmetric SM extensions, models with excited leptons, with heavy neutrinos, with a heavy fermion triplet, and many others. Figure 9 (right) shows the results from the ATLAS search for excited electrons and muons in the $p p \rightarrow l l^{*} \rightarrow l l \gamma$ channel, assuming the excited leptons are produced via contact interactions [74]. The limits derived based on data recorded at $\sqrt{s}=8 \mathrm{TeV}$ and corresponding to $13 \mathrm{fb}^{-1}$ integrated luminosity are converted into lower bounds on the compositeness scale $\Lambda$.

Although in many NP models the DM particle interacts so weakly that it cannot be observed directly, the requirement of the correct relic density can place significant constraints on some theoretically motivated models [75]. A prime example is a scenario with gravitino DM: late-time gravitino production in decays of a heavier particle is typically accompanied by the release of large amounts of electromagnetic and hadronic energy, capable of spoiling the predictions of the Big Bang Nucleosynthesis. Stringent bounds on model parameters can also be obtained for scenarios with an axino or a right-handed scalar neutrino as DM candidate.

The number of different NP models put forth to date is so large that it would be impossible to confront separately each such model with experimental data. Therefore, one is often forced to treat the SM as an effective field theory (EFT) and parametrise the (lack of) knowledge of NP by higher-dimensional operators. This approach is used, in particular, for mono- $X$ searches of DM at the LHC. However, if the NP scale $\Lambda$ is not too far away from the electroweak scale, such an approach can lead to too strong bounds on $\Lambda$, as it was demonstrated in [76] in the context of a very simple SM extension and its EFT.

\section{Outlook}

The next run of the LHC at a c.m. energy of $13 \mathrm{TeV}$ will allow for more detailed analyses and extend the reach to higher physics scales. It will push the precision on electroweak physics and provide further insights in the underlying theory of particle physics.

\section{Acknowledgments}

The authors would like to thank the organizers of DIS2014 for the perfect organization and the friendly and fruitful atmosphere of the workshop.

\section{References}

[1] G. Aad et al. [ATLAS Collaboration], Observation of a new particle in the search for the Standard Model Higgs boson with the ATLAS detector at the LHC, Phys. Lett. B 716 (2012) 1 [arXiv:1207.7214 [hep-ex]]; G. Aad et al. [ATLAS Collaboration], ATLAS-CONF-2012-162.

[2] S. Chatrchyan et al. [CMS Collaboration], Observation of a new boson at a mass of $125 \mathrm{GeV}$ with the CMS experiment at the LHC Phys. Lett. B 716 (2012) 30 [arXiv:1207.7235 [hep-ex] ];

S. Chatrchyan et al. [CMS Collaboration], CMS-PAS-HIG-12-045. 
[3] C. Englert, A. Freitas, M. Muhlleitner, T. Plehn, M. Rauch, M. Spira and K. Walz, Precision Measurements of Higgs Couplings: Implications for New Physics Scales, arXiv: 1403.7191 [hep-ph].

[4] D. Buttazzo, G. Degrassi, P. P. Giardino, G. F. Giudice, F. Sala, A. Salvio and A. Strumia, Investigating the near-criticality of the Higgs boson, JHEP 1312 (2013) 089 [arXiv: 1307.3536 ]; G. Degrassi, S. Di Vita, J. Elias-Miro, J. R. Espinosa, G. F. Giudice, G. Isidori and A. Strumia, Higgs mass and vacuum stability in the Standard Model at NNLO, JHEP 1208 (2012) 098 [arXiv:1205.6497 [hep-ph]].

[5] O. Buchmueller, M. J. Dolan, J. Ellis, T. Hahn, S. Heinemeyer, W. Hollik, J. Marrouche and K. A. Olive et al., Implications of Improved Higgs Mass Calculations for Supersymmetric Models, Eur. Phys. J. C 74 (2014) 2809 [arXiv:1312.5233 [hep-ph] ].

[6] G. Aad et al. [ATLAS Collaboration], Measurement of the Higgs boson mass from the $H \rightarrow \gamma \gamma$ and $H \rightarrow Z Z^{*} \rightarrow 4 \ell$ channels with the ATLAS detector using $25 \mathrm{fb}^{-1}$ of pp collision data, arXiv:1406.3827 [hep-ex].

[7] The CMS Collaboration, Precise determination of the mass of the Higgs boson and studies of the compatibility of its couplings with the standard model, CMS-PAS-HIG-14-009.

[8] A. David et al. [LHC Higgs Cross Section Working Group Collaboration], LHC HXSWG interim recommendations to explore the coupling structure of a Higgs-like particle, arXiv: 1209.0040 [hep-ph].

[9] S. Chatrchyan et al. [CMS Collaboration], Search for the standard model Higgs boson produced in association with a W or a Z boson and decaying to bottom quarks, Phys. Rev. D 89 (2014) 012003 [arXiv:1310.3687 [hep-ex]].

[10] S. Chatrchyan et al. [CMS Collaboration], Evidence for the direct decay of the 125 GeV Higgs boson to fermions, Nature Phys. 10 (2014) [arXiv:1401.6527 [hep-ex] ].

[11] The ATLAS Collaboration, Search for the bb decay of the Standard Model Higgs boson in associated (W/Z)H production with the ATLAS detector, ATLAS-CONF-2013-079.

[12] The ATLAS Collaboration, Evidence for Higgs Boson Decays to the tau+tau-Final State with the ATLAS Detector, ATLAS-CONF-2013-108.

[13] The ATLAS Collaboration, Updated coupling measurements of the Higgs boson with the ATLAS detector using up to 25/fb of proton-proton collision data, ATLAS-CONF-2014-009.

[14] G. Aad et al. [ATLAS Collaboration], Search for the Standard Model Higgs boson decay to $\mu^{+} \mu^{-}$ with the ATLAS detector, arXiv:1406.7663 [hep-ex] .

[15] The CMS Collaboration, Search for the standard model Higgs boson in the $\mu^{+} \mu^{-}$decay channel in pp collisions at $\sqrt{s}=7$ and $8 \mathrm{TeV}$, CMS-PAS-HIG-13-007.

[16] The CMS Collaboration, https://twiki.cern.ch/twiki/bin/view/CMSPublic/ttHCombinationTwiki.

[17] The ATLAS Collaboration, earch for the Standard Model Higgs boson produced in association with top quarks and decaying to $b \bar{b}$ in pp collisions at $\sqrt{s}=8 \mathrm{TeV}$ with the ATLAS detector at the LHC, ATLAS-CONF-2014-11.

[18] V. Khachatryan et al. [CMS Collaboration], Constraints on the Higgs boson width from off-shell production and decay to Z-boson pairs, Phys. Lett. B 736 (2014) 64, [arXiv: 1405.3455 [hep-ex]]. 
[19] The ATLAS Collaboration, Determination of the off-shell Higgs boson signal strength in the high-mass ZZ final state with the ATLAS detector, ATLAS-CONF-2014-042.

[20] G. Aad et al. [ATLAS Collaboration], Search for the neutral Higgs bosons of the Minimal Supersymmetric Standard Model in pp collisions at $\sqrt{s}=7$ TeV with the ATLAS detector, JHEP 1302 (2013) 095 [arXiv: 1211.6956 [hep-ex]].

[21] S. Chatrchyan et al. [CMS Collaboration], Search for a Higgs boson decaying into a b-quark pair and produced in association with b quarks in proton-proton collisions at 7 TeV, Phys. Lett. B 722 (2013) 207 [arXiv:1302.2892 [hep-ex]].

[22] The CMS Collaboration, Higgs to tau tau (MSSM), CMS-PAS-HIG-13-021.

[23] The ATLAS Collaboration, Search for Higgs bosons in Two-Higgs-Doublet models in the $H \rightarrow W W \rightarrow$ evmuv channel with the ATLAS detector, ATLAS-CONF-2013-027.

[24] The ATLAS Collaboration, Constraints on New Phenomena via Higgs Coupling Measurements with the ATLAS Detector, ATLAS-CONF-2014-10.

[25] The CSM Collaboration, Search for extended Higgs sectors in the $H \rightarrow$ hh and $A \rightarrow$ Zh channels in $\sqrt{s}=8 \mathrm{TeV}$ pp collisions with multileptons and photons final states, CMS-PAS-H IG-13-025.

[26] The ATLAS Collaboration, Search for a Higgs boson decaying to four photons through light CP-odd scalar coupling using $4.9 \mathrm{fb}-1$ of $7 \mathrm{TeV}$ pp collision data taken with ATLAS detector at the LHC, ATLAS-CONE-2012-079.

[27] The CMS Collaboration, Search for a non-standard-model Higgs boson decaying to a pair of new light bosons in four-muon final states, CMS-PAS-HIG-13-010.

[28] The ATLAS Collaboration, Search for charged Higgs bosons in the $\tau+$ jets final state with pp collision data recorded at $\sqrt{s}=8 \mathrm{TeV}$ with the ATLAS experiment, ATLAS-CONF-2013-090.

[29] G. Aad et al. [ATLAS Collaboration], Search for a light charged Higgs boson in the decay channel $H^{+} \rightarrow c \bar{s}$ in $t \bar{t}$ events using pp collisions at $\sqrt{s}=7$ TeV with the ATLAS detector, Eur. Phys. J. C 73 (2013) 2465 [arXiv:1302.3694 [hep-ex]].

[30] The CMS Collaboration, Search for the light charged Higgs boson in top quark decays in pp collisions at $\sqrt{s}=7 \mathrm{TeV}$, CMS-HIG-11-019.

[31] S. Chatrchyan et al. [CMS Collaboration], Search for a light charged Higgs boson in top quark decays in pp collisions at $\sqrt{s}=7 \mathrm{TeV}$, JHEP 1207 (2012) 143 [arXiv:1205.5736 [hep-ex]].

[32] G. Aad et al. [ATLAS Collaboration], Search for doubly-charged Higgs bosons in like-sign dilepton final states at $\sqrt{s}=7 \mathrm{TeV}$ with the ATLAS detector, Eur. Phys. J. C 72 (2012) 2244 [arXiv:1210.5070 [hep-ex]].

[33] G. Aad et al. [ATLAS Collaboration], Search for Invisible Decays of a Higgs Boson Produced in Association with a Z Boson in ATLAS, Phys. Rev. Lett. 112 (2014) 201802 [arXiv: 1402.3244 [hep-ex]].

[34] The ATLAS Collaboration, Search for flavour changing neutral currents in top quark decays $t \rightarrow c H$, with $H \rightarrow \gamma \gamma$, and limit on the tcH coupling with the ATLAS detector at the LHC, ATLAS-CONF-2013-081.

[35] The CMS Collaboration, Search for a heavy Higgs boson in the H to ZZ to 2l2nu channel in pp collisions at $\sqrt{s}=7$ and $8 \mathrm{TeV}$, CMS-PAS-HIG-13-014. 
[36] S. Chatrchyan et al. [CMS Collaboration], Search for a Higgs boson decaying into a $Z$ and a photon in pp collisions at $\sqrt{s}=7$ and 8 TeV, Phys. Lett. B 726 (2013) 587 [arXiv: 1307.5515 [hep-ex]].

[37] The CMS Collaboration, Search for a Higgs boson decaying into gamma*gamma to mu+mu-gamma with dilepton mass below $20 \mathrm{GeV}$ in pp collisions at $\sqrt{\mathrm{s}}=8 \mathrm{TeV}$, CMS-PAS-HIG-2014-033.

[38] S. Chatrchyan et al. [CMS Collaboration], Search for invisible decays of Higgs bosons in the vector boson fusion and associated ZH production modes, arXiv: 1404.1344 [hep-ex] .

[39] The CMS Collaboration, Search for the resonant production of two Higgs bosons in the final state with two photons and two bottom quarks in pp collisions at $\sqrt{s}=8 \mathrm{TeV}$. CMS-PAS-HIG-13-032.

[40] G. Aad et al. [ATLAS Collaboration], Search for Higgs Boson Pair Production in the $\gamma \gamma b \bar{b}$ Final State using pp Collision Data at $\sqrt{s}=8$ TeV from the ATLAS Detector, arXiv:1406.5053 [hep-ex] .

[41] R. Santos, The CP-conserving 2HDM after the $8 \mathrm{TeV}$ run, these proceedings.

[42] G. Aad et al. [ATLAS Collaboration], Evidence for the spin-0 nature of the Higgs boson using ATLAS data, Phys. Lett. B 726 (2013) 120 [arXiv:1307.1432 [hep-ex] ]; Study of the spin of the new boson with up to $25 \mathrm{fb}^{1}$ of ATLAS data, ATLAS-CONF-2013-040.

[43] S. Chatrchyan et al. [CMS Collaboration], Measurement of Higgs boson production and properties in the WW decay channel with leptonic final states, JHEP 1401 (2014) 096 [arXiv : 1312.1129 [hep-ex]].

[44] S. Chatrchyan et al. [CMS Collaboration], Measurement of the properties of a Higgs boson in the four-lepton final state, Phys. Rev. D 89 (2014) 092007 [arXiv: 1312.5353 [hep-ex] ].

[45] V. Sanz, talk at DIS2014, Higgs Spin and CP at the LHC.

[46] A. Djouadi, W. Kilian, M. Muhlleitner and P. M. Zerwas, Production of neutral Higgs boson pairs at LHC, Eur. Phys. J. C 10 (1999) 45 [hep-ph/ 9904287$].$

[47] J. Baglio, A theoretical review of triple Higgs coupling studies at the LHC in the Standard Model, these proceedings; A. Massironi, Higgs Pair Production via Vector Boson Fusion at the LHC, these proceedings.

[48] R. Contino, M. Ghezzi, C. Grojean, M. Muhlleitner and M. Spira, eHDECAY: an Implementation of the Higgs Effective Lagrangian into HDECAY, arXiv:1403.3381 [hep-ph] .

[49] R.S. Gupta, BSM Primary Effects: The complete set of predictions from the dimension-6 BSM Lagrangian, These proceedings.

[50] The ATLAS Collaboration,

https://atlas.web.cern. ch/Atlas/GROUP S / PHYS ICS / CombinedSummaryPlots / SM/index.html\#ATLAS_e_SMSummary_FiducialRatio.

[51] The ATLAS Collaboration, Measurement of the $W+W$-production cross section in proton-proton collisions at $\sqrt{s}=8 \mathrm{TeV}$ with the ATLAS detector, ATLAS-CONF-2014-033; S. Chatrchyan et al. [CMS Collaboration], Measurement of $W+W$ - and ZZ production cross sections in pp collisions at $\operatorname{sqrt}(s)=8 \mathrm{TeV}$, Phys. Lett. B 721 (2013) 190 [arXiv:1301.4698 [hep-ex]].

[52] T. Kasprzik, Electroweak corrections to vector-boson pair production at the LHC: Monte Carlo implementation \& NNLO contributions, these proceedings.

[53] M. Rauch, Multi-boson Production in Weak Boson Fusion, these proceedings. 
[54] M. Luszczak, Production of $W^{+} W^{-}$pairs via subleading processes at the LHC, these proceedings.

[55] G. Aad et al. [ATLAS Collaboration], Measurement of the electroweak production of dijets in association with a Z-boson and distributions sensitive to vector boson fusion in proton-proton collisions at $\sqrt{s}=8 \mathrm{TeV}$ using the ATLAS detector, JHEP 1404 (2014) 031 [arXiv: 1401.7610 [hep-ex] ]; The CMS Collaboration, Measurement of pure electroweak production of a $Z$ boson in association with two forward/backward jets in proton-proton collisions at $8 \mathrm{TeV}$, CMS-PAS-FSQ-12-035.

[56] S. Chatrchyan et al. [CMS Collaboration], A search for $W W \gamma$ and $W Z \gamma$ production and constraints on anomalous quartic gauge couplings in pp collisions at $\sqrt{s}=8 \mathrm{TeV}$, arXiv:1404.4619 [hep-ex].

[57] G. Aad et al. [ATLAS Collaboration], Search for supersymmetry at $\sqrt{s}=8 \mathrm{TeV}$ in final states with jets and two same-sign leptons or three leptons with the ATLAS detector, JHEP 1406 (2014) 035 [arXiv:1404.2500 [hep-ex]].

[58] The ATLAS Collaboration, Search for Supersymmetry in Diphoton Events with Large Missing Transverse Momentum in $8 \mathrm{TeV}$ pp Collision Data with the ATLAS Detector, ATLAS-CONF-2014-001.

[59] The ATLAS Collaboration, A general search for new phenomena with the ATLAS detector in $p p$ collisions at $\sqrt{s}=8 \mathrm{TeV}$, ATLAS-CONF-2014-006.

[60] S. Chatrchyan et al. [CMS Collaboration], Search for new physics in the multijet and missing transverse momentum final state in proton-proton collisions at $\sqrt{s}=8 \mathrm{TeV}$, JHEP 1406 (2014) 055 [arXiv:1402.4770 [hep-ex]].

[61] V. Khachatryan et al. [CMS Collaboration], Searches for electroweak production of charginos, neutralinos, and sleptons decaying to leptons and $\mathrm{W}, \mathrm{Z}$, and Higgs bosons in pp collisions at $8 \mathrm{TeV}$, arXiv:1405.7570 [hep-ex].

[62] The CMS Collaboration, Search for electroweak production of charginos and neutralinos in final states with a Higgs boson in pp collisions at $\sqrt{s}=8 \mathrm{TeV}$, CMS-PAS-SUS-13-017.

[63] The CMS Collaboration, Search for electroweak production of higgsinos in channels with two Higgs bosons decaying to b quarks in pp collisions at $8 \mathrm{TeV}$, CMS-PAS-SUS-13-022.

[64] G. Aad et al. [ATLAS Collaboration], Search for direct production of charginos, neutralinos and sleptons in final states with two leptons and missing transverse momentum in pp collisions at $\sqrt{s}=8$ TeV with the ATLAS detector, JHEP 1405 (2014) 071 [arXiv: 1403.5294 [hep-ex] ].

[65] K. I. Hikasa and M. Kobayashi, Light Scalar Top at e+e-Colliders, Phys. Rev. D 36 (1987) 724.

[66] M. Muhlleitner and E. Popenda, Light Stop Decay in the MSSM with Minimal Flavour Violation, JHEP 1104 (2011) 095 [arXiv: 1102.5712 [hep-ph] ].

[67] C. Boehm, A. Djouadi, Y. Mambrini, Decays of the lightest top squark, Phys. Rev. D61 (2000) 095006 [hep-ph/9907428].

[68] The ATLAS Collaboration, https: //atlas.web.cern.ch/Atlas/GROUPS/PHYSICS/CombinedSummaryPlots/ SUSY/index.html\#ATLAS_SUSY_Stop_tLSP.

[69] The CMS Collaboration, https://twiki.cern.ch/twiki/bin/view/CMSPublic/SUSYSMSSummaryPlots8TeV. 
[70] E. Popenda, Squark Production and Decay at NLO matched with Parton Showers, these proceedings.

[71] Sophie Henrot-Versillé, Constraining Supersymmetry using the relic density and the Higgs boson, these proceedings.

[72] Béranger Dumont, Higgs coupling measurements and impact on the MSSM, these proceedings.

[73] V. Khachatryan et al. [CMS Collaboration], Search for massive resonances decaying into pairs of boosted bosons in semi-leptonic final states at $\sqrt{s}=8 \mathrm{TeV}$, arXiv:1405.3447 [hep-ex] .

[74] G. Aad et al. [ATLAS Collaboration], Search for excited electrons and muons in $\sqrt{s}=8 \mathrm{TeV}$ proton-proton collisions with the ATLAS detector, New J. Phys. 15 (2013) 093011 [arXiv:1308.1364 [hep-ex]].

[75] Ki-Young Choi, Non-neutralino dark matter, these proceedings.

[76] Giorgio Busoni, On the Validity of the Effective Field Theory for Dark Matter Searches at the LHC, these proceedings. 